\title{
Therapie mit Weihrauch bei Morbus Crohn
}

Autor: Henning Gerhardt

\section{ZUSAMMENFASSUNG}

Die Studie „Therapie des aktiven Morbus Crohn mit dem Boswelliaserrata-Extrakt H15“ [3] erbrachte den Nachweis, dass eine Therapie mit Boswellia-serrata-Extrakt $\mathrm{H} 15$ der Behandlung des aktiven Morbus Crohn mit Mesalazin nicht unterlegen ist. Bei gleichzeitiger Berücksichtigung der Verträglichkeit ist im Sinne einer Nutzen-Risiko-Bewertung der Boswellia-serrata-Extrakt H15 günstiger zu bewerten als Mesalazin. Die Studie PS0201B0 [4] bestätigt die gute Verträglichkeit des neuen Boswellia-serrata-Extraktes Boswelan ${ }^{\circledR}$ in der Langzeitbehandlung. Leider konnte in dieser Studie nicht einmal eine Tendenz bezüglich einer Wirksamkeit nachgewiesen werden. Daher ist davon auszugehen, dass Weihrauch zur Remissionserhaltung bei Morbus Crohn nicht wirksam ist.

Schlüsselwörter

Weihrauch, Mesalazin, Morbus Crohn, Verträglichkeit,

Remissionserhaltung.

\section{ABSTRACT}

The study „Therapie des aktiven Morbus Crohn mit dem Boswellia-serrata-Extrakt H15“" (Therapy of Active Crohn's Disease with the Boswellia Serrata Extract H15) [3] provided evidence, that a therapy with the Boswellia serrata extract $\mathrm{H} 15$ is not inferior to the treatment of active Crohn's disease with mesalazine. When the tolerance is also taken into consideration, the Boswellia serrata extract $\mathrm{H} 15$ has to be assessed more favorable than mesalazine in the sense of a benefit-risk assessment. The study PS0201B0 [4] confirms the tolerance of the new Boswellia serrata extract Boswelan ${ }^{\circledR}$ in the long-term treatment. Unfortunately, in this study it was not possible to prove a tendency regarding an effectiveness. Therefore, it must be assumed that incense is not effective for the remission maintenance with Crohn's disease.

Keywords

Incense, mesalazine, Crohn's disease, tolerance, remission maintenance.

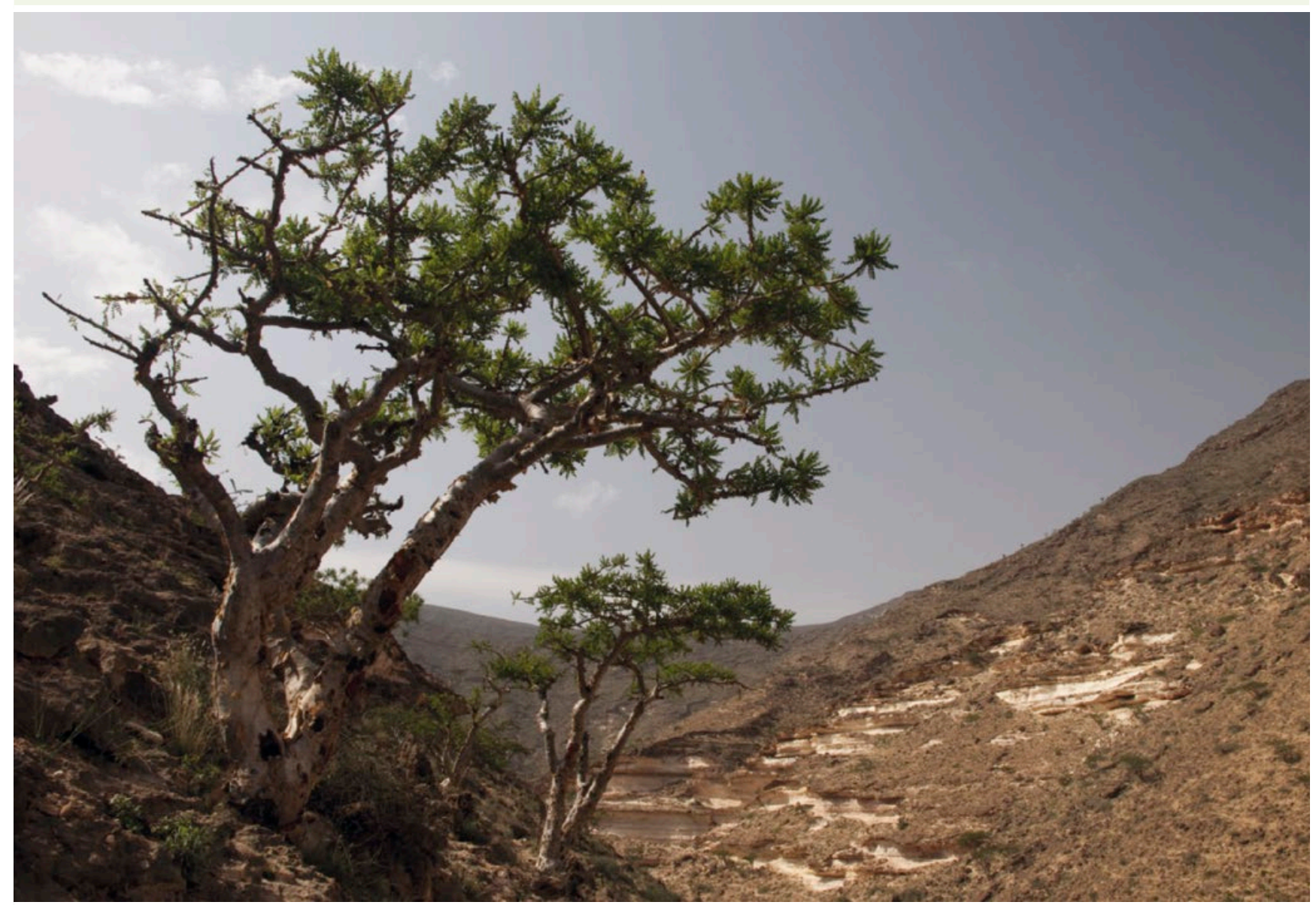

Abb. 1 Der indische Weihrauchbaum liefert mit seinem Harz den Grundstoff für den medizinischen Einsatz von Weihrauch. @ Maroš Markovič/ Fotolia 


\section{Therapeutische Erfahrungen}

Die Therapieerfahrungen mit Boswellia-serrata-Extrakt H15/Sallaki-Gufic bei chronisch-entzündlichen Darmerkrankungen (CED) stützen sich seit 1993 auf die schulmedizinischen Therapieansätze und Leitlinien sowie auf die komplementäre Therapie mit H15 bei Patienten mit chronisch-entzündlichen Erkrankungen - speziell mit den chronisch entzündlichen Darmerkrankungen Morbus Crohn und Colitis ulcerosa.

\section{Anfangsdosierung}

Zu der zumeist bei Therapiebeginn bestehenden Standardtherapie mit Kombinationen von Salizylaten, Kortikoiden und Immunsuppressiva wird zusätzlich in der Regel mit einer Dosis von 3-mal 2 Tabletten H15/Sallaki der Firma Gufic à $400 \mathrm{mg}(2,4 \mathrm{~g})$ - rezeptpflichtig - begonnen. Ziel war es, die bestehende Kortikoidtherapie mit ihren zahlreichen unerwünschten Arzneimittelwirkungen (UAW) auszuschleichen.

Das Präparat H15 Gufic ist ein Gesamtextrakt aus dem Harz des indischen Weihrauchbaumes Boswellia serrata. Einer seiner Inhaltsstoffe, die Boswelliasäuren, wirken als spezifischer 5-Lipoxygenasehemmer und vermindern die Entstehung von Leukotrienen und damit die zelluläre Immunreaktion.

\section{Einnahme}

H15-Tabletten werden vor oder nach dem Essen eingenommen. Wir empfehlen, die Tabletten vor dem Schlucken zu zerbeißen oder sie in ein Trinkglas mit einem Fingerbreit Leitungswasser zu geben. Im Wasser zerfallen die Tabletten in ca. 20 min, vor dem Trinken sollte der Patient das Glas einmal kurz schwenken.

\section{Verträglichkeit}

H15 Gufic wird von den Patienten gut vertragen. Nur wenige beklagen den eigentümlichen Weihrauchgeschmack. Dieser kann durch die Einnahme in Form von Gelatinekapseln vermieden werden. Oberbauchbeschwerden wurden kaum berichtet. Sehr selten traten allergische Exantheme meist in Verbindung mit einem Infekt auf. Bei jeweils negativer dermatologischer Austestung setzten die Patienten die begonnene H15-Therapie fort.

Kortisonbedingte unerwünschte Arzneimittelwirkungen, wie Fresssucht mit zwangsläufiger Gewichtszunahme mit „Mondgesicht“ und „Stiernacken“, Steroid-Diabetes, Stimmungsschwankungen bis hin zur Depression, dünne Haut und Osteoporose, wurden bei unseren Patienten unter der Boswelliatherapie bisher nicht beobachtet. Es traten deutlich weniger Erkältungsinfekte in den Wintermonaten auf.

\section{Medikamentenreduktion}

Bei spürbarem Wirkungseintritt der H15-Gufic-Therapie mit Nachlassen der Bauchschmerzen und Bauchkrämpfe haben wir die Patienten angehalten, ihre Vormedikation bei konstantem Wohlbefinden wöchentlich um eine Tablette zu reduzieren. Dabei konnten wir in der Regel mit dem Kortison beginnen und über eine Reduzierung der Dosierung von $20 \mathrm{mg}$ auf $15 \mathrm{mg}$ auf $10 \mathrm{mg}$ und $10 \mathrm{mg}$ jeden zweiten Tag das Kortison schließlich absetzen; anschließend die Salizylate. Schließlich wurde auch die H15Monotherapie nach 4 bis 6 Wochen langsam reduziert und eine $\mathrm{H} 15$-Erhaltungstherapie-Dosis ermittelt.

\section{Akuter Schub}

Im Falle eines akuten Schubes der Grunderkrankung mit erhöhten Entzündungsparametern (BKS, CRP) reagierten wir mit einer Erhöhung der H15-Dosierung, z. B. 3-mal 3 Tabletten (3,6 g). Falls diese H15-Dosiserhöhung nicht ausreichte, sprachen CED-Patienten, die zuvor einige Monate kortisonfrei geworden waren, auf eine Gabe von z. B. $30 \mathrm{mg}$ Prednisolon/d über 3 bis 5 Tage meist sehr gut an.

\section{Weitere Studien zur Therapie mit Weihrauch}

\section{Nachweis der Wirksamkeit und Unbedenklichkeit}

Um die Wirksamkeit und Unbedenklichkeit der H15-Therapie zu beweisen, führten wir die Studie „Therapie des aktiven Morbus Crohn mit dem Boswellia-serrata-Extrakt H15“ durch, deren Ergebnisse in der Z Gastroenterol 2001 erschienen sind [3]. Der Nachweis, dass H15 der Therapie mit Mesalazin nicht unterlegen ist, kann als Wirksamkeitsnachweis nach dem Stand der Wissenschaft bei der Behandlung des aktiven Morbus Crohn mit dem Boswellia-serrata-Extrakt bewertet werden, da die Wirksamkeit von Mesalazin bei dieser Indikation entsprechend der Zulassung als erwiesen gilt. Bei gleichzeitiger Berücksichtigung der Verträglichkeit ist im Sinne einer Nutzen-RisikoBewertung der Boswellia-serrata-Extrakt H15 günstiger zu bewerten als Mesalazin. Die Ergebnisse zur Wirksamkeit und Unbedenklichkeit bestätigen die Testhypothese, dass H15 in Sinne einer Nutzen-Risikobewertung dem Mesalazin nicht unterlegen ist.

\section{Studien zur Remissionserhaltung}

Mit der Studie PS0201B0 [4] beauftragte die Firma Pharmasan Prof. Holtmeier aus Köln-Porz, das neue Präparat Boswelan ${ }^{\circledR}$ auf seine Wirksamkeit und Sicherheit in einer Langzeittherapie von 52 Wochen an in Remission befindlichen MC-Patienten zu evaluieren. Das Präparat ist ein von Dr. Elias Müller entwickeltes Weihrauchpräparat, das die schonende Extraktion von Boswellia serrata in Kapseln 
enthält. Die Studie bestätigt in der Zusammenfassung die gute Verträglichkeit des Boswellia-serrata-Extrakts in der Langzeitbehandlung. Leider konnte nicht einmal eine Tendenz bezüglich einer Wirksamkeit nachgewiesen werden, daher ist davon auszugehen, dass Weihrauch zur Remissionserhaltung beim Morbus Crohn nicht wirksam ist.

\section{Unbedenklichkeit und Sicherheit der H15-Therapie}

Wegen der guten Verträglichkeit von Boswellia serrata und besonders des Präparats H15 Gufic und seiner engen therapeutischen Verwandtschaft zu den Kortikosteroiden drängen sich folgende Fragen aus dem Nebenwirkungsspektrum der Kortikoide zur Prüfung für eine Langzeittherapie auf:

\section{Wie steht es um die Organtoxizität?}

Buvari beschreibt in ihrer Dissertation „Wirksamkeit und Unbedenklichkeit der H15-Ayurmedica-Therapie bei chronisch-entzündlichen Erkrankungen“ [2] bei 2057 Patienten, die bis zu 6 Jahren mit H15 Gufic behandelt wurden, einen unauffälligen laborchemischen Verlauf unter dieser Therapie. Einzig den signifikanten Rückgang des Hämoglobins bei CED-Patienten erklärt sie mit einem „DilutionEffekt“, der nur bei CED-Patienten, nicht aber bei Rheumapatienten zu beobachten war.

\section{Wie steht es um die Verträglichkeit?}

Seifert beschreibt in seiner Dissertation „Gesundheitsbezogene Lebensqualität bei chronisch entzündlichen Erkrankungen“ im Rahmen der Therapiestudie „Therapie des aktiven Morbus Crohn mit Boswellia-serrata-Extrakt H15“ [5], die Verbesserung der Lebensqualität u. a. im sf-36 in allen 8 Items signifikant gegenüber der Mesalazin-Vergleichsgruppe, die nur in 3 Items vergleichbar waren.

\section{Welche Auswirkungen hat Boswellia serrata auf Osteo- porose?}

Bouhmidi-Boumariz beschreibt in ihrer Dissertation „Veränderung der Knochendichte bei der Therapie mit dem Boswellia-serrata-Extrakt $\mathrm{H} 15$ bei chronisch entzündlichen Darmerkrankungen “ [1] Zunahmen der Knochendichte und weniger Schmerzen bei CED-Patienten, die unter Boswellia-serrata-Extrakt H15 kortisonfrei wurden.

\section{Gibt es eine teratogene Wirkung?}

Eine teratogene Wirkung ist für Boswellia serrata, das Präparat H15 Gufic und für andere Boswellia-serrata-Anwendungen nicht bekannt. Auf Nachfrage der Medizinischen Ethikkommission II der Universität Heidelberg am Universitätsklinikum Mannheim erhielten wir vom Hersteller die Auskunft, dass H15 Gufic in Indien gegen Erbrechen in der Schwangerschaft erfolgreich eingesetzt würde und dabei keine „Side Effets“ bekannt geworden seien.

\section{Gibt es eine tumorstatische Wirkung}

Die tumorhemmende Wirkung des hochdosierten Boswellia-serrata-Extrakts H15 durch Hemmung der Topoisomerasen ist belegt. Auch in der hohen Dosierung ist $\mathrm{H} 15$ gut verträglich und bereitet den Patienten - gegenüber anderen zugelassenen Topoisomerasehemmern - keine unerwünschten Arzneimittelwirkungen.

\section{Briefe von Patienten aus der Praxis}

Die Wirksamkeit und gute Verträglichkeit von Weihrauch bei Morbus Crohn wurde mir im Laufe der Jahre immer wieder durch meine Patienten bestätigt, wie folgende Briefauswahl aus den Jahren 2016-2017 zeigt.

\section{Fall 1: Frau J. H., geboren 1987, MC, Brief an Dr. Gerhardt vom 17.01.2017}

Erstmalig bekam ich die Diagnose Morbus Crohn mit 21 Jahren, Ende 2009. Die ersten 2 Jahre reichte es mir, Pentasa zu nehmen, damit es mir gut ging. Ich hinterfragte das Medikament nicht - es half gegen meine Durchfälle. 2012, ich hatte gerade mein Studium beendet und mich über meinen ersten Job gefreut, kam der große Einbruch. Ich hatte so starke und blutige Durchfälle, dass ich in kürzester Zeit gut 10 Kilo abnahm und nur noch ein Schatten meines Selbst war. Meine damalige Gastroentrologin kommentierte meinen Zustand mit den Worten: „Sie sind die schwerste Patientin, die ich seit Jahren habe. Sie werden ihr Leben lang auf starke Medikamente angewiesen sein. “ Erst brach eine Welt für mich zusammen. Ich stand am Scheideweg meines Lebens. Entweder ich würde diese Nachricht akzeptieren und mich in Zukunft mit Kortison, Pentasa, Budenofalk und im schlimmsten Fall Azathioprin behandeln lassen oder ich würde einen Weg finden, um mich selbst zu heilen. Ich ging nie wieder zu dieser Ärztin.

Mein erster Schritt bestand darin, im Internet zu forschen, welche Medikamente es auf pflanzlicher Basis gibt. Schnell fand ich heraus, dass man mit Weihrauch gute Erfahrungen gemacht hatte. Kurzerhand kontaktierte ich Dr. Gerhardt. Ich erzählte ihm meine Geschichte. Er machte mir Mut und verschrieb mir Weihrauch. Ich nahm diesen über Monate in der höchsten Dosierung. Und er half. Ich möchte hier nicht von Zauberei sprechen. Man nimmt nicht Weihrauch und ist schlagartig gesund. Viele Faktoren gehören dazu, dass man wieder gesund wird: Ein gesunder Geist, der Glaube daran, dass alles gut wird, eine ausgewogene Ernährung. Auch dies hat mir Dr. Gerhardt vor Augen geführt. Es hat bei mir insgesamt fast 8 Jahre gedauert bis ich von mir sagen konnte: ich bin wieder gesund. Eine kürzlich durchgeführte Darmspiegelung zeigt deutlich, dass ich keinen Morbus Crohn mehr habe und die minimal unter dem Mikroskop sichtbaren Entzündungen lediglich einer sehr leichten Colitis indeterminata zuzuordnen sind. Heute nehme ich gar keine Medikamente mehr. Auch keinen Weihrauch. Für diesen Zustand musste ich 
hart kämpfen und mich immer wieder aufrappeln. Dabei half mir Weihrauch enorm, weil er keine Nebenwirkungen auslöste und ich mir sicher sein konnte, dass ich keine Chemie zu mir nehme, sondern ein Naturprodukt. Das war für mich von größter Wichtigkeit, weil ich meine zuvor eher ungesunde Ernährung radikal umgestellt hatte. Ich esse seit Jahren schon sehr viel Obst und Gemüse, wenig glutenhaltige Nahrungsmittel und kaum Milchprodukte, alles in Bioqualität. Seit ungefähr 9 Monaten nehme ich zusätzlich Wildkräutersmoothies zu mir, die wohl den letzten Schritt zur Selbstheilung ausgelöst haben.

Abschließend möchte ich zusammenfassen, dass ich ohne Weihrauch in der Spirale der chemischen Medikamente versunken wäre. Das hätte dazu geführt, dass ich immer auf diese Medikamente angewiesen gewesen wäre und ich mich nicht durch meine Ernährung selbst hätte heilen können. Denn eins ist für mich gewiss, ich brauche mich nicht biologisch und rohköstlich zu ernähren, wenn ich meinen Körper gleichzeitig durch Medikamente mit Chemie verseuche.

Fall 2: Herr R. G, geboren 1974, MC ED (1/99), Brief an Dr. Gerhardt vom 3.9.2016

Mein WEG war und ist Weihrauch, gesunde Ernährung und positive Gedanken. Seit fast 20 Jahren (1997) gibt mir die Einnahme von Weihrauch das gute Gefühl, etwas für mich und meine Gesundheit zu tun - ohne Risiken und Nebenwirkungen. In der ganzen Zeit sind keine akuten Schübe (bei Morbus Crohn) mehr aufgetreten. Auch wenn es vereinzelt immer wieder verwunderte Ärzte beim Thema „Medikation Weihrauch“ gibt, ist und bleibt dieser mein treuer Begleiter und wird von mir immer wieder wärmstens empfohlen!

Fall 3: Frau B. W., geboren 1972, MC, cP, IAST, Brief an Dr. Gerhardt vom 3.12.2016

Als meine Mutter mir den Vorschlag machte, ein Weihrauchpräparat mit dem gewöhnungsbedürftigen Namen „H15“ zu probieren, war ich nur wenig begeistert: Noch eine Tablette mehr, dachte ich, und dazu eine, von der man Wirkung und Nebenwirkung kaum kennt. Niemand in meinem Umfeld hatte Erfahrung mit diesem Medikament. Insgesamt überwog die Skepsis, denn mein Crohn war zu dieser Zeit kein Leichtgewicht: Innerhalb von fünf Jahren fünf große Bauch-OPs, davon eine Notfall-Operation, Fistelbildung, Ileostoma-Anlage, zwei Thrombosen, eine Osteoporose mit Wirbelbrüchen durch die Kortison-Therapie, eine Crohn-Manifestation an der Haut - wirklich Ruhe hatte ich eigentlich nie. Zudem stand eine sechste Operation aus, bei der wieder einmal eine Fistel entfernt werden sollte. Nachdem ich mich näher mit den Wirkprinzipien der Boswelliasäuren beschäftigt hatte, kam ich jedoch zu dem Schluss, es mit dem Medikament zu probieren.
Zuerst zeigte sich aus meiner Sicht nur wenig Erfolg: Die Fistel blieb, die Operation musste also sein. Nach der Operation wurde ich dann eines Besseren belehrt. Nur 20 Zentimeter meines Darms waren noch vom Crohn befallen, der Rest schien „gesund“ zu sein. Von der OP erholte ich mich schnell und setzte nach kurzer Zeit alle Medikamente - bis auf den Weihrauch-Extrakt - ab. Die erste schubfreie Zeit seit Langem! Und sie hielt fast 10 Jahre an. In dieser Zeit nahm ich relativ regelmäßig die Weihrauchtabletten. Immer, wenn ich das Gefühl hatte, der Crohn könnte sich melden, nahm ich wieder eine Weile das Präparat. Bis sich leider wieder ein Schub einstellte - wie gewöhnlich mit Bauchschmerzen und Gewichtsabnahme. Zunächst schien es mit Kortison, Azathioprin und Boswellia wieder bergauf zu gehen, nach einer Kur fühlte ich mich wieder fit - bis eine Fistel kam. Ich erhöhte die Weihrauchdosis und zu meiner absoluten Überraschung schloss sich die Fistel wieder! Leider nur für 3 Monate, für mich aber genug Zeit, um einen schönen Urlaub zu machen. Nach zwei weiteren Operationen bin ich inzwischen wieder seit 10 Jahren beschwerdefrei: Ich habe das Gefühl den Crohn im Griff zu haben - und nicht der Crohn mich. Wahrscheinlich eine Nebenwirkung der Boswelliasäure.

Fall 4: Frau O. M., geboren 1971, MC, Begleitrheumatismus, Brief vom 19.4.2017

Heute möchte ich über meine Erfahrung mit Weihrauch seit 1998 berichten. Zu meiner Vorgeschichte: Erstdiagnose M. C. 8/1987, mehrfache Schübe seitdem, Therapie mit Sulfasalazin (bei rheumatischen Beschwerden), Mesalazin (zum Erhalt bzw. zur Verlängerung der schubfreien Zeiten), Prednison bzw. Prednisolon und Methylprednisolon im akuten Schub oder auch länger (mal mit Gabe von Calcium, später auch ohne!).

Die Wirkung: Mesalazin bewirkte keine Verbesserung im Vergleich zu Zeiten ohne Medikation, Azulfidine linderte die rheumatischen Beschwerden etwas, die ersten Gaben von Prednison und Prednisolon sowie Methylprednisolon zeigten eine signifikante Verbesserung der Beschwerden. Nach Ausschleichen der Therapie flammten die Entzündungen aber immer wieder auf. Es folgte eine Langzeitgabe verbunden mit starken Nebenwirkungen, gegen die zusätzliche Medikamente erforderlich wurden. Mit jeder neuen Behandlung eines Schubes mit Glukokortikoiden ließ die positive Wirkung des Wirkstoffes nach. Sonstige Beschwerden in Verbindung mit MC außerhalb des Darmes (außer rheumatoide Symptomatik): Erythema nodosum, Uveitis, Stomatitis aphtosa, Migräne - kein Medikament half gegen diese Beschwerden - häufiges Auftreten auch unter Medikation. Damit ging eine stark verminderte Lebensqualität und Leistungsfähigkeit einher. Zu den Nebenwirkungen: Wassereinlagerungen, Mondgesicht, starke Akne, unter Prednison Depression, Verlust der Libido, Magenschmerzen. 5/1996 - Medikation wie zuvor - immer 
wieder Auftreten der Symptomatik mit Bauchkrämpfen, Durchfall und Verstopfung im Wechsel.

Seit 1998 Einnahme von Weihrauch (H15 und Sallaki) sowie indische Flohsamenschalen. Wirkung: Es zeigte sich eine schnelle und deutliche Verbesserung ohne Nebenwirkungen. Selbst die Beschwerden außerhalb des Darmes (vor allem die rheumatoiden Beschwerden, Entzündungen der Haut, Augen und im Mund sowie die Migräne waren deutlich weniger bis gar nicht mehr vorhanden). Zu Beginn der Therapie glaubte ich nicht an die Wirkung, da ich nur allzu oft von Medizinern belächelt wurde, wenn ich davon berichtete. Trotz meiner eigenen Zweifel, nahm ich das Medikament weiter ein, zudem ich keine Nebenwirkungen feststellen konnte. Ich stellte hingegen fest, dass ich bei akuten Beschwerden zumeist eine sehr rasche Wirkung im Sinne einer Linderung der Schmerzen und Verbesserung der Darmtätigkeit erkennen konnte. Einschlägig zeige sich mir diese Wirkung durch folgendes Erlebnis: Ich erwachte morgens gegen sechs Uhr mit starken Bauchschmerzen, in aller Verzweiflung nahm ich zwei Tabletten auf einmal, kaute sie und behielt den Brei für einige Zeit im Mund. Noch während ich am Tisch saß und mit dem Brei im Mund aus dem Fenster schaute, fragte ich mich plötzlich, weshalb ich überhaupt aufgestanden war. Da fiel mir wieder ein, dass ich doch Bauchschmerzen gehabt hatte. Ich konnte diese glücklicherweise nicht mehr wahrnehmen - ging zurück ins Bett und schlief entspannt wieder ein.

Dennoch zweifelte ich, auch viele Ärzte waren der Meinung, dieses Erlebnis sei ein Zufall oder Einbildung gewesen. Da sich ähnliche Vorfälle jedoch wiederholten, führte ich die Therapie mit Weihrauch fort. Leider wurde um das Jahr 2000 die Teilzulassung für Weihrauch zurückgenommen und ich musste das Medikament selbst bezahlen was für mich fast nicht möglich war. Nur in den schlimmsten Beschwerdephasen „gönnte“ ich mir Weihrauch, verzichtete dafür auf vieles andere.

Ende April 2010 durchlitt ich einen erneuten Schub, erhielt Kortison und Mesalazin mit mäßiger Wirkung. Damals war ich stationär in Behandlung im Stadtkrankenhaus in Frankenthal, wo ich auch zögerlich von Weihrauch berichtete. Zu meinem Erstaunen fanden sich dort Mediziner, die dessen Wirkung kannten, mir das Medikament jedoch nicht auf Kassenrezept verordnen konnten. Ich selbst sagte mir, wenn die Krankenkasse Weihrauch nicht als Kassenleistung anerkennt, müsse das daran liegen, dass die anderen Medikamente besser wirken. So nahm ich mehr als ein halbes Jahr Kortison ein. Im Juli/August 2010 erfolgte jeweils eine Dilatation. Im Oktober 2016 ließ die Wirkung des Kortisons immer mehr nach und die Dosis wurde in der Folge immer höher - die Dilatation war nur von kurzem Erfolg bzgl. der Stenose, die Entzündung flammte erneut auf. In meiner Verzweiflung kaufte ich mir Weihrauch und konnte nach einer ca. 4- bis 5-wöchigen Ausschleichphase das Kortison unter hausärztlicher Kontrolle absetzen. Mesala- zin hatte ich bereits mit Beginn der Einnahme des Weihrauchs abgesetzt. Die Erhaltungsphase der symptomfreien Zeit ohne Kortison unter Weihrauchtherapie ohne die genannten Nebenwirkungen zeige sich über einige Monate. Selbst nach Absetzen des Weihrauchs aus Kostengründen blieb ich noch einige Wochen beschwerdefrei. Ende 2011 erfolgte eine erneute Dilatation, ich verweigerte jedoch jede Medikation, es sei denn, man hätte mir Weihrauch verordnet, denn meine Erfahrung mit der „Leitlinienmedikation" war eine sehr ungute gewesen. Warum sollte ich mir eine solche Belastung antun? Nur, weil sie gesetzlich erlaubt ist und auf lange Sicht eine volkswirtschaftliche Ersparnis bedeutet?

Im Mai 2012 durchlitt ich einen erneuten schweren Schub, ich nahm trotzdem an einem Wochenendausflug in einer anderen Stadt teil, wo ich vom Hotel direkt ins Krankenhaus musste. Dort erfolgte eine weitere, von großen Schmerzen begleitete Diagnostik (infolge 2-maliger Vorbereitung auf eine Koloskopie bei bestehender Stenose im Bereich des neoterminalen lleums) und von den Leitlinien gekrönte Behandlung. Diese half mir über das Gröbste hinweg. Ich glaubte an diese Leitlinien, weil sie von Fachleuten geschrieben worden waren. Ca. 4 Wochen später kam ich in eine Anschlussheilbehandlung zur Gewichtszunahme für den Fall, dass man mich doch erneut operieren müsste (bei knapp $44 \mathrm{~kg}$ bei 1,65 cm Köpergröße). Ein Zeichen dafür, dass die behandelnden Ärzte von der unsicheren Wirkung der „Leitlinienbehandlung“ wissen mussten. In der Klinik der Heilbehandlung erfuhr ich, dass man inzwischen von der Langzeittherapie mit Kortison abraten würde und für eine Langzeittherapie andere, nebenwirkungsärmere Immunsuppressiva empfahl. Also begann ich eine Therapie mit Azathioprin. Die Ärzte sagten mir, wenn die Wirkung nicht nach spätestens 6 Monaten einträte, sei dies kein Medikament, das bei mir wirken würde. Man müsse dann andere Wirkstoffe ausprobieren. Ich ließ mich auf die Behandlung ein - der Preis dafür war eine sehr große Abwehrschwäche. Eine Wirkung trat leider nicht ein, auch nicht auf die sonstigen Beschwerden, die mich von Zeit zu Zeit wieder quälten. Im April 2013 entschloss ich mich, Azathioprin unter ärztlicher Überwachung abzusetzen und besorgte mir wieder Weihrauch.

Interessenkonflikte

Der Autor gibt an, dass kein Interessenkonflikt besteht. 


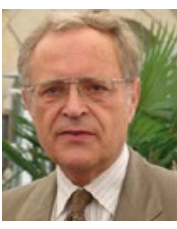

\section{Dr. med. Henning Gerhardt}

Henning Gerhardt ist Facharzt für Innere Medizin mit Zusatzbezeichnung Gastroenterologie und war von 1979-2008 als Oberarzt der Medizinischen Kliniken der Medizinischen Fakultät Mannheim der Rupprecht-Karls-Universität Heidelberg tätig. 1979 gründete er die Colitis-Crohn-Ambulanz und die Deutsche Colitis-Crohn-Vereinigung Selbsthilfegruppe Rhein-Neckar (DCCV SHG-Rhein-Neckar) an der Universitätsmedizin Mannheim (UMM). Seit 2008 berät er Patienten mit Autoimmunerkrankungen privatärztlich.

\section{Korrespondenzadresse}

Dr. med. Henning Gerhardt

Arzt für Innere Medizin

Am Oberen Luisenpark 32

68165 Mannheim

E-Mail: dr.henninggerhardt@gmx.de
[1] Bouhmidi-Boumariz Z. Veränderung der Knochendichte bei der Therapie mit dem Boswellia serrata Extrakt $\mathrm{H} 15$ bei chronisch entzündlichen Darmerkrankungen [Dissertation]. Ruprecht-Karls-Universität Heidelberg: Fakultät für Klinische Medizin Mannheim; 2004

[2] Buvari P. Wirksamkeit und Unbedenklichkeit der H15-Ayurmedica-Therapie bei chronisch-entzündlichen Erkrankungen [Dissertation]. Ruprecht-Karls-Universität Heidelberg: Fakultät für Klinische Medizin Mannheim; 2006

[3] Gerhardt H, Seifert F, Buvari P et al. Therapie des aktiven Morbus Crohn mit Boswellia-serrata-Extrakt H15. Z Gastroenterol 2001; 39: 11-17

[4] Holtmeier W, Zeuzem S, Preiss ] et al. Randomized, placebo-controlled, double-blind trial of Boswellia serrata in maintaining remission of Crohn's disease: good safety profile but lack of efficacy. Inflamm Bowel Dis 2011; 17: 573-582

[5] Seifert F. Gesundheitsbezogene Lebensqualität bei chronisch entzündlichen Erkrankungen [Dissertation]. RuprechtKarls-Universität Heidelberg: Fakultät für Klinische Medizin Mannheim; 2004

Bibliografie

DOI https://doi.org/10.1055/s-0043-112405

EHK 2017; 66: 166-171

(c) MVS Medizinverlage Stuttgart GmbH \& Co. KG ISSN 0014-0082 